\title{
Organizational Excellence
}

Fresno State is committed to developing our employees by providing training and development opportunities that will help participants LEARN best practices, IMPROVE processes, SHARE best practices and CELEBRATE accomplishments

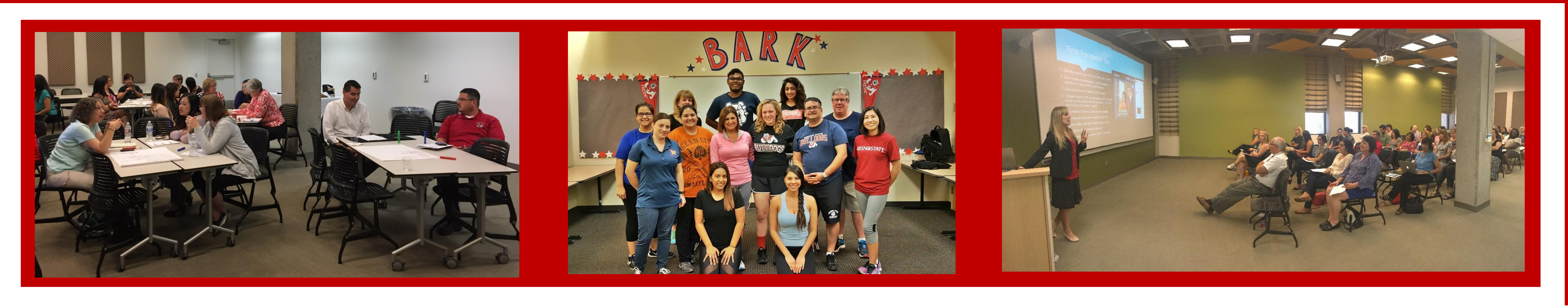

Creativity and Innovation for Effectiveness: CAIFE 2.0 CAIFE is a unique organizational development program that engages faculty, staff fand
administratrors in the procecss of institutional transformation. CAlFE strengthens the administrators in the processs of institutitonal transte
culture of creativity and innovation at tresso ostate

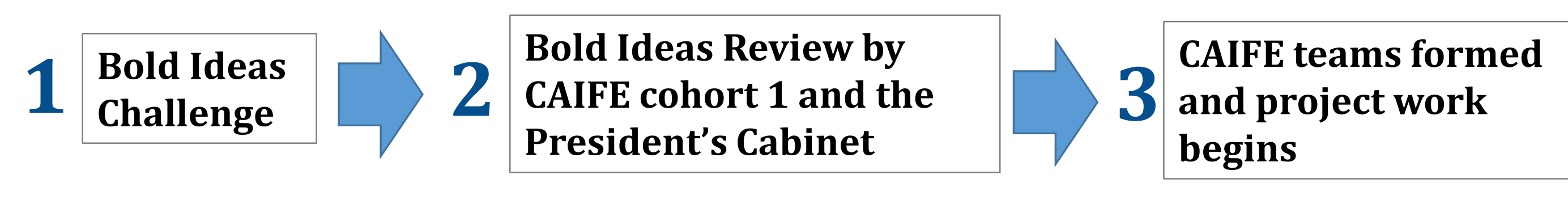
Supporting Learning and Development In 2016/17 we offered nearly 400 workshops to for faculty, staff, managers and department chairs.

Track
Navigating Fresno State
Developing A Healthy U
Lunch \& Learn
Enhancing Your Technical Skills
Leadership Skills for Managers \&
Department Chairs

\# of Workshops \# \# Attendees

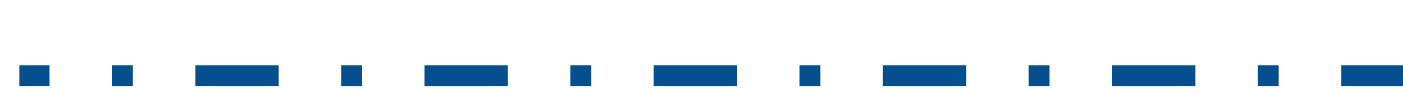

STAR Day - May 25, 2017 "Not All Heroes Wear Capes" Join us in celebrating our SUPER staff at this year's STAR (Staff Training And Recognition) Day

Workshops will center around the theme, Everyone is encouraged to dress as their favorite superhe department!

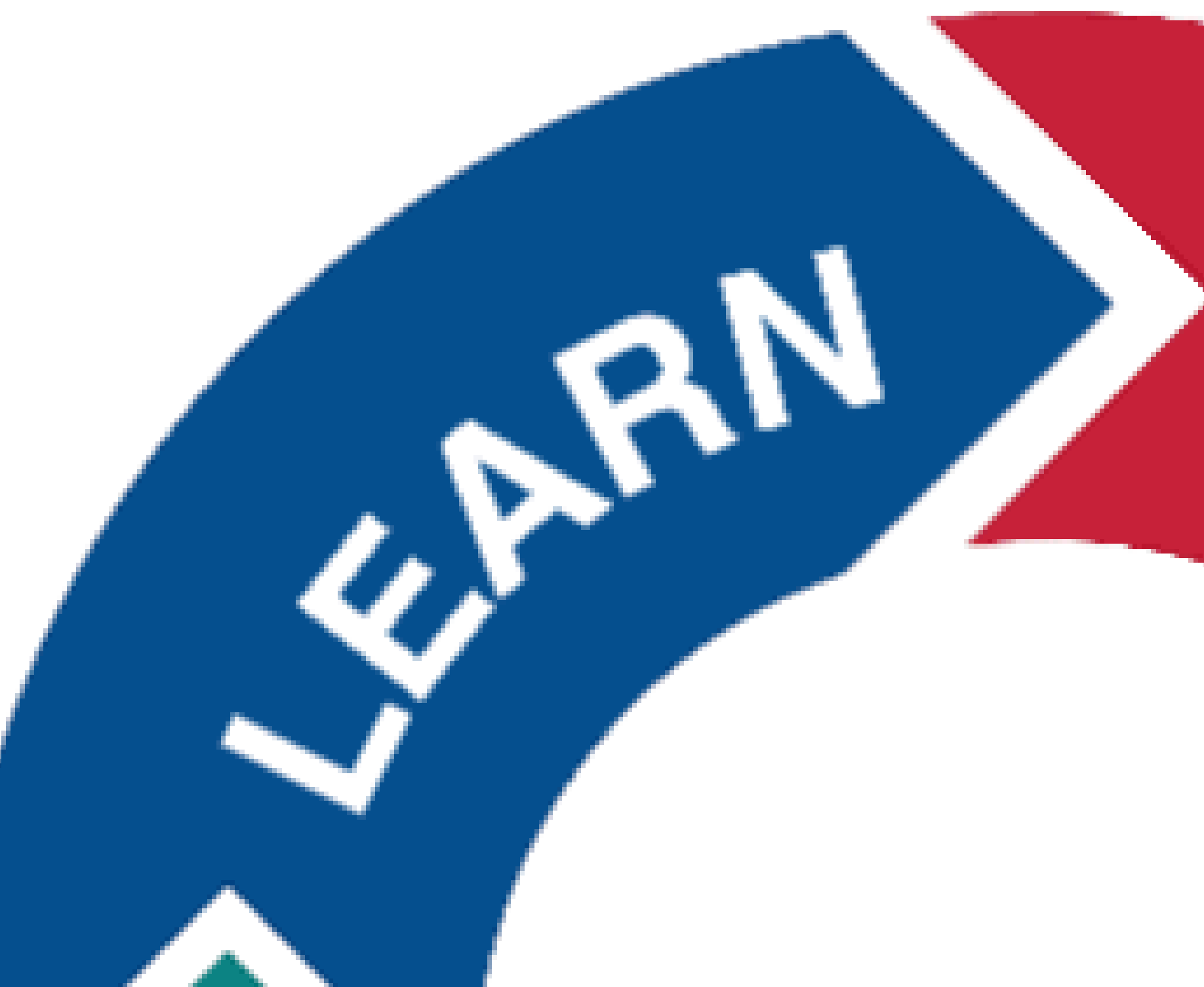

9 CAIFE teams (73 participants representing faculty, staff and students)
have worked throughout 2016/17 to implement the Bold Ideas.

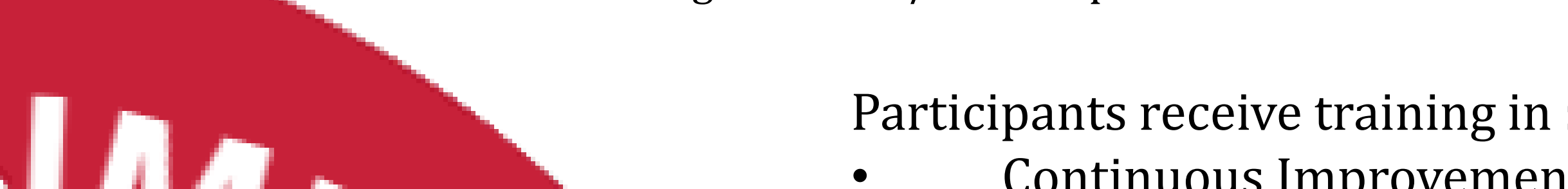

$1 M$ Innovation Change Management

personal and professional achievements at their closing session. Participants attended monthly meetings from
September to April and learned about Fresno State, effective communication strategies, work-life balance, and more. The program focused on networking and building relationships across campus. 45 employees
completed the Administrative Academy in 2016/17.

izational Development Enhancing Work Group interactions Organizational Excellence helps with team building, focus
groups retreat planning and facilitation, custom workshops and much more.

017

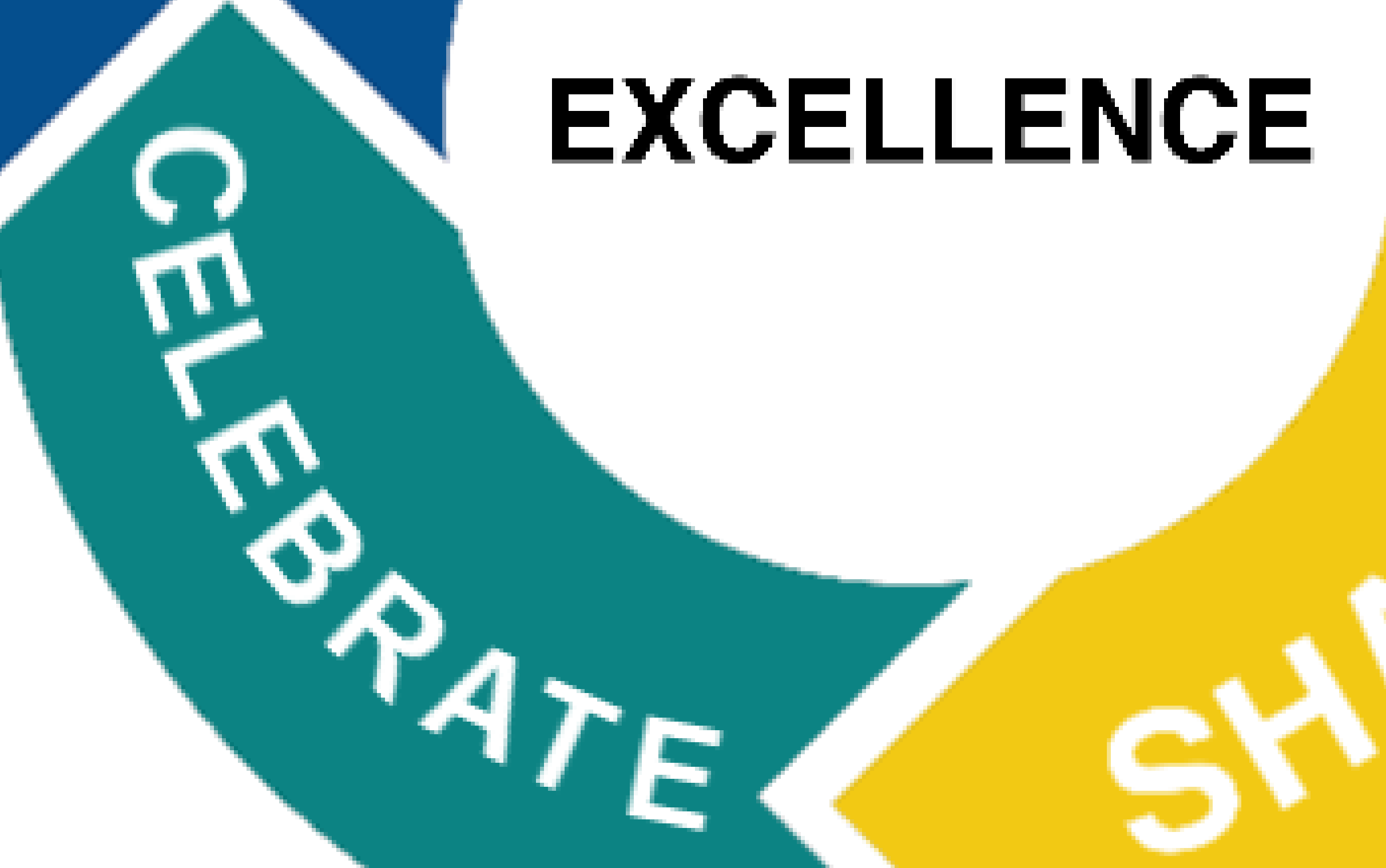

ellence

President's Showcase of Excellence

This year marks the $3^{\text {rd }}$ annual President's Showcase of Excellence and the first year it is open to the
community. Nearly 100 posters sharing stories of innovation, excellence, and achievement from across Fresno State's campus are featured at this year's Showcase.

Join us for the President's Toast to Excellence at
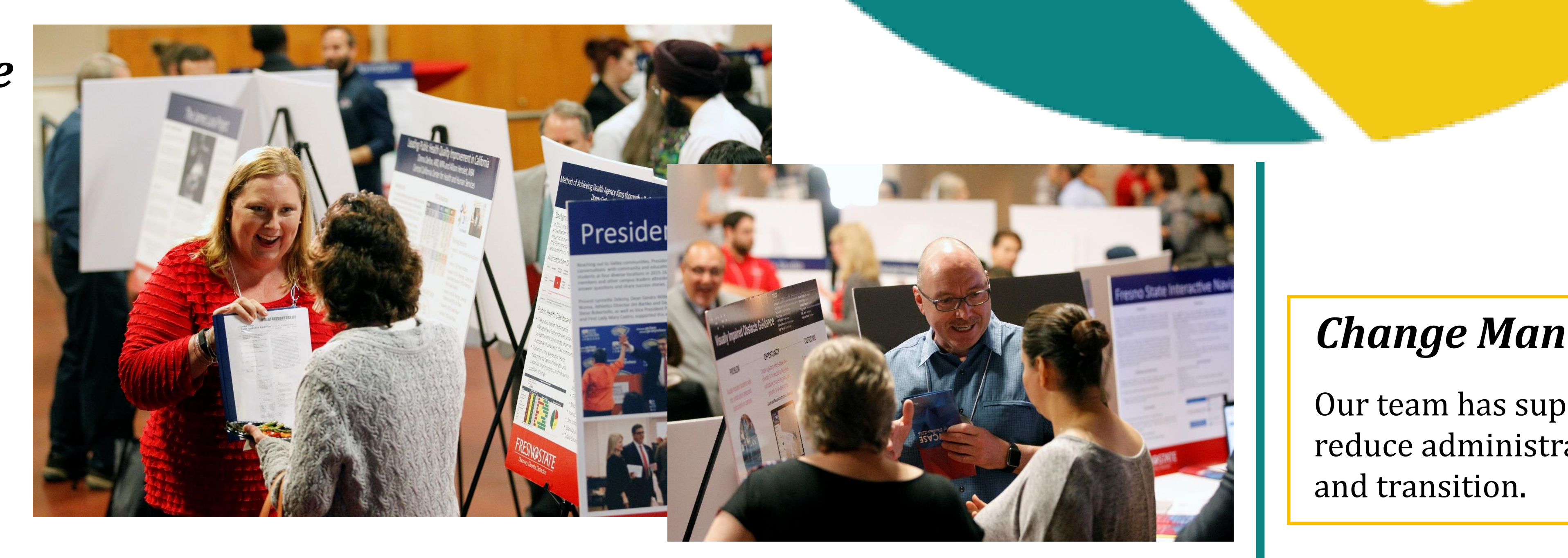

\section{Faculty and Staff Mentor Program 22 faculty and staff were paired with seasoned campus leaders as part of Fresno State's first joined faculty-staff mentor cohort. This program is co-sponsored with Faculty Affairs and the Mentor
Institute. Applications for 2017/18will be available this summe}

\section{Change Management \& Continuous Improvement}

Our team has supported numerous continuous improvement projects to reduce administrative burden, improve efficiencies and support change and transition.

\section{FRESNQSTATE}

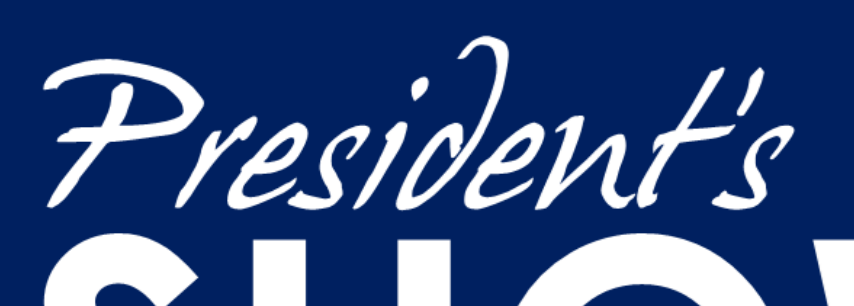

SHOWCASE

of Excellence 2017 